\title{
Tool material surface alloying by wide-aperture low-energy high-current electron beam treatment before wear-resistant coating
}

\author{
Sergey N. Grigoriev ${ }^{a}$ And Sergey V. Fedorov \\ Moscow State University of Technology "STANKIN", 1 Vadkovsky per., 127994 Moscow GSP-4, Russian Federation
}

Received 24 September 2015, Accepted 2 October 2015

\begin{abstract}
The alloying of surfaces of tool materials by wide-aperture low-energy high-current electron beam before wear-resistant coating is developed. This technology makes possible to enlarge a lifetime of cutting tools. The regularities of high-melting compounds formation in the near-surface layer are established. The high-melting compounds are obtained by reaction of self-spreading high-temperature synthesis. The microstructure of modified surface layers is investigated by optical microscopy.
\end{abstract}

Key words: Tool materials / surface modification / self-propagating high-temperature synthesis / low-energy high-current electron beam / cutting tools

\section{Introduction}

Currently, there are many ways to improve the properties of metal-cutting tools through surface doping. The most widespread are methods of chemical heat treatment. An alternative to the traditional methods of chemical heat treatment can be the creation of a doped surface layer of micron thickness with improved wear resistance on steel or hardmetal tools with the use of low-energy highcurrent electron beam (LHEB). In some cases, LHEB in addition to melting and mixing of the surface layers of the target initiates the reaction of self-propagating high-temperature synthesis (SHS), which is implemented through the use of internal energy of the chemical interaction of the initial reagents. The practice of cutting shows that the tools with such treatment in complex with sequentially applied ion-plasma resistant coating meet the highest requirements for quality, reliability and productivity [1]. Due to the modified near-surface layer changes the mechanism of tool wear, significantly reduces cutting force, which, in turn, is beneficial to the tool resource.

\section{Methodology of research}

When creating an SHS system quite a large range of reactive at high temperature substances [2] can be used as reagents. At the same time other substances can be used as fillers or diluents, including the ones participating in the synthesis as side-reaction products. It's important to

${ }^{a}$ Corresponding author: science@stankin.ru provide conditions for effective interaction of the reactants, in particular exothermicity. An example is the synthesis reaction with the formation of nitride and carbide phases and reactions between decomposing compounds with elements of the basis.

In the experiments were used 2 types of base materials: plates of hard alloy H13A (Sandvik Coromant) and plates of high-speed steel P6M5, pre-nitrided in a two-step vacuum-arc discharge at a depth of about $50 \mu \mathrm{m}$ [3]. The samples were covered by thin coat of $\mathrm{Zr}$ with thickness from 150 to $250 \mathrm{~nm}$ by means of magnetron to obtain crystals $\mathrm{ZrN}$ in the surface layer in case of high-speed steel and a coat of the NbHfTi alloy in the case of processing of the hard alloy to get a layer of wear-resistant non-stoichiometric carbides. Then, eliminating the contact with the air, in the same process chamber the samples were subjected to a series of pulses of wide-aperture LHEB, which, by heating the surface to a high temperature, thereby initiating chemical reactions both in liquid and solid phases between the metal cover, nitrogen and carbon, comprising the basis, both in free form or in compounds.

The processing was carried out with the use of the unit "RITM-SP" (produced by "Microsplav", Tomsk), which is a combination of the source of LHEB and several magnetron sputtering systems located in the same vacuum chamber (Fig. 1). The installation allows deposition of coats of different materials on the surface of the desired product and subsequent liquid-phase mixing of the materials of the coat and LHEB substrate in a single vacuum cycle [4]. As a result of this treatment, the coating with 


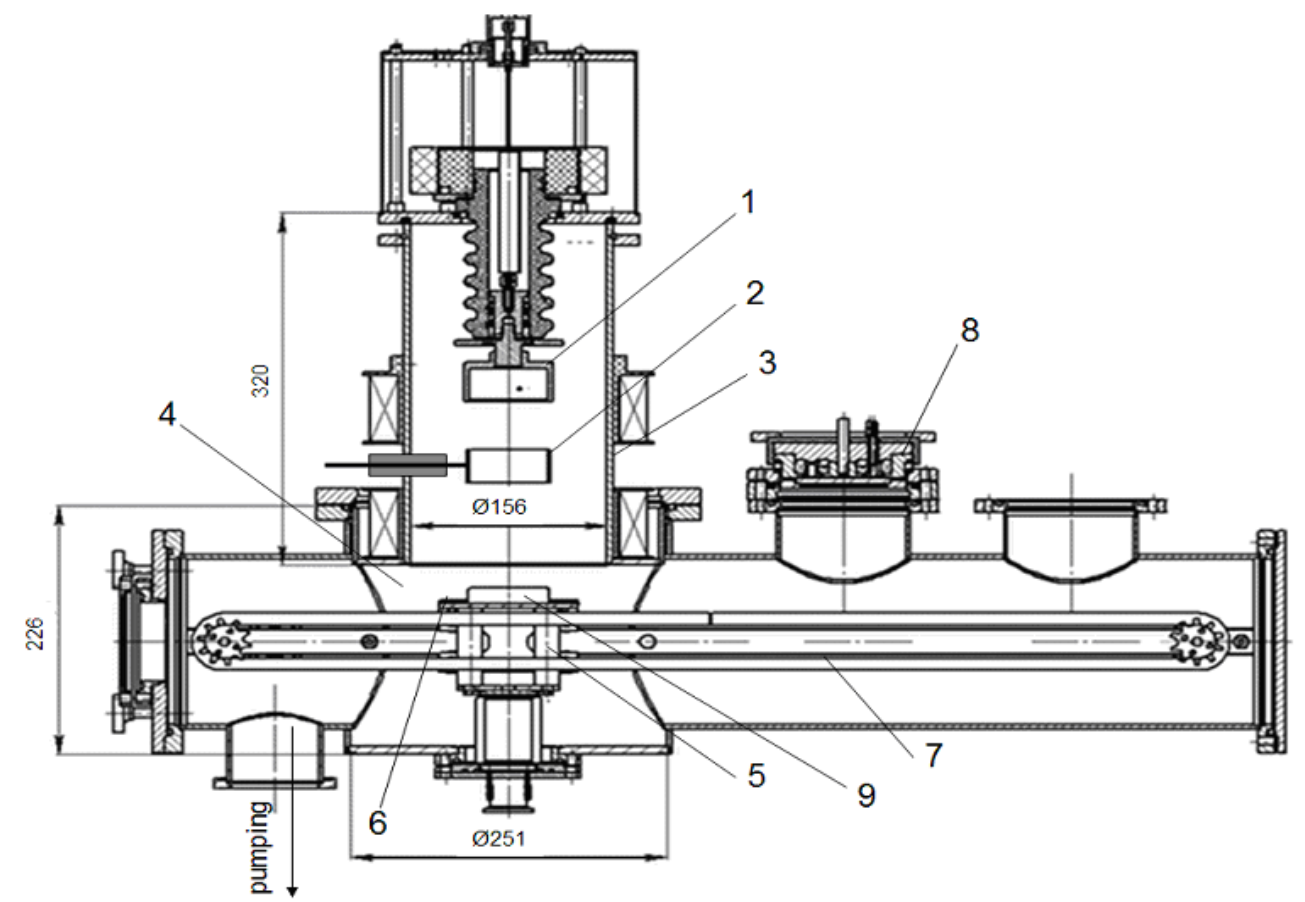

Fig. 1. Layout of the unit "RITM-SP". 1 - explosive emission cathode, 2 - anode, 3 - the case of electron gun, 4 - working chamber, 5-copper sleeve, 6 - carriage, 7 - chain, 8 - magnetron, 9 - processed object [4].

a given chemical composition is deposited on the surface of the sample and fused in it.

In this case the sharp interfacial boundary between the coat and the substrate is eroded and appears an extended transition layer (several micrometer thickness) with varying elemental composition, thereby achieving the highest level of adhesion of the coating to the substrate, which was previously demonstrated in many metallic systems coat-substrate [5].

LHEB generation occurs by extracting electrons from the cathode plasma produced due to explosive electrical issue on metal cathode microtips and their subsequent acceleration in the double electric layer. Further the beam is transported in the plasma-filled diode to the collector. Use of such circuit of generation and transportation allows receiving a beam of microsecond duration with a current density up to $104 \mathrm{~A} / \mathrm{cm}^{2}$ and energy of electrons 15-30 keV on a collector. The area of one-time processing of a target, equal to the diameter of a beam, can be up to $100 \mathrm{~cm}^{2}$.

\section{Results of research}

SHS in thin coats is initiated when the temperature in the surface layer of the sample exceeds a certain level. In case of high heat losses due to the intense heat removal in the substrate the process may lose stability in short terms. Eventually, however, the process is stabilized, because with the increasing of surface temperature SHS front velocity grows exponentially. The assessment of temperature conditions, irradiated by LHEB, shows that the surface temperature reaches values much higher than the melting point of the components. The appearance of the melt in the amount of more refractory particles leads to a sharp increase of the interfacial surface and growth of the speed of a chemical reaction between the components.

Figure 2 demonstrates the data illustrating the result of exposure of a series of several LHEB pulses with energy density of $4.5 \mathrm{~J} . \mathrm{cm}^{-2}$ and duration of about $5 \mu \mathrm{s}$ to the surface of the nitrided sample of steel P6M5. The figure shows that the irradiation of the thin $(0.2 \mu \mathrm{m}) \mathrm{Zr}$ coat previously applied to the sample using magnetron spray diffuser, leads to the initiation of the chemical reaction of formation of refractory $\mathrm{ZrN}$ in the surface layer with thickness of about $2 \mu \mathrm{m}$. This occurs because the electron-beam treatment causes dissociation of iron nitrides, especially $\varepsilon$-phase.

Formation of a nitride phase is confirmed by data of the X-ray diffraction analysis of the sample processed by LHEB which is presented in Figure 3. The diffraction pattern shows accurate zirconium nitride peaks along with phases of $\alpha$-and $\gamma$-iron.

Figure 4 shows data illustrating the result of LHEB impact on the plate of carbide H13A. On the fracture are clearly visible two distinct layers - surface and intermediate. The concentration of $\mathrm{Nb}, \mathrm{Hf}$ and $\mathrm{W}$ in the intermediate layer indicates the mixing of the solid alloy with the alloy coating by reaction of high-temperature synthesis. Thickness, within which it is possible to obtain a modified SHS structure, is 3-4 $\mu \mathrm{m}$. Multiple initiating of LHEB process practically does not alter the original microstructure. To make the transformation complete, typically, a series of five or six LHEB pulses is enough. 


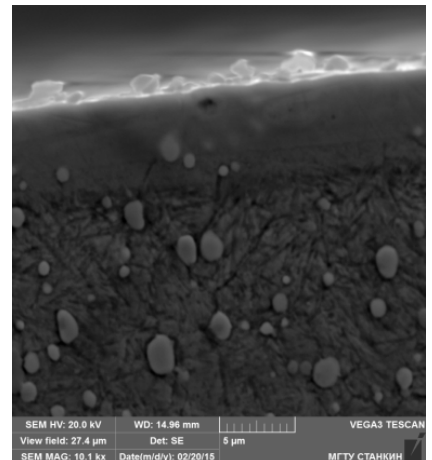

(a)

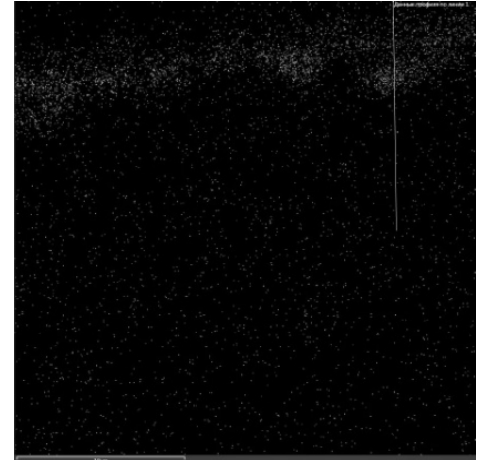

(b)

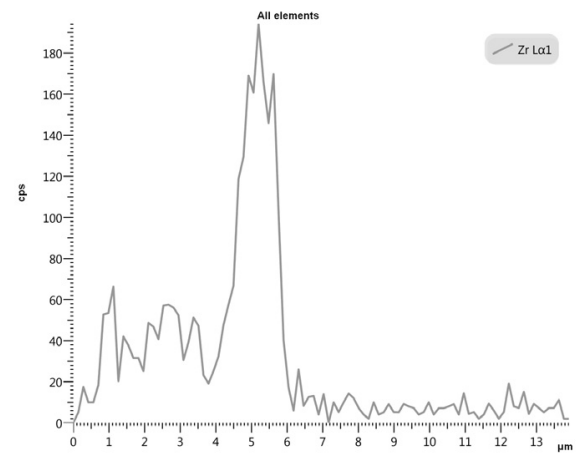

(c)

Fig. 2. Modification with zirconium nitride surface layer of high-speed steel P6M5: (a) SEM image of the etched section, (b) map of the distribution of zirconium on the section, (c) Zr profile data on the scanning line.

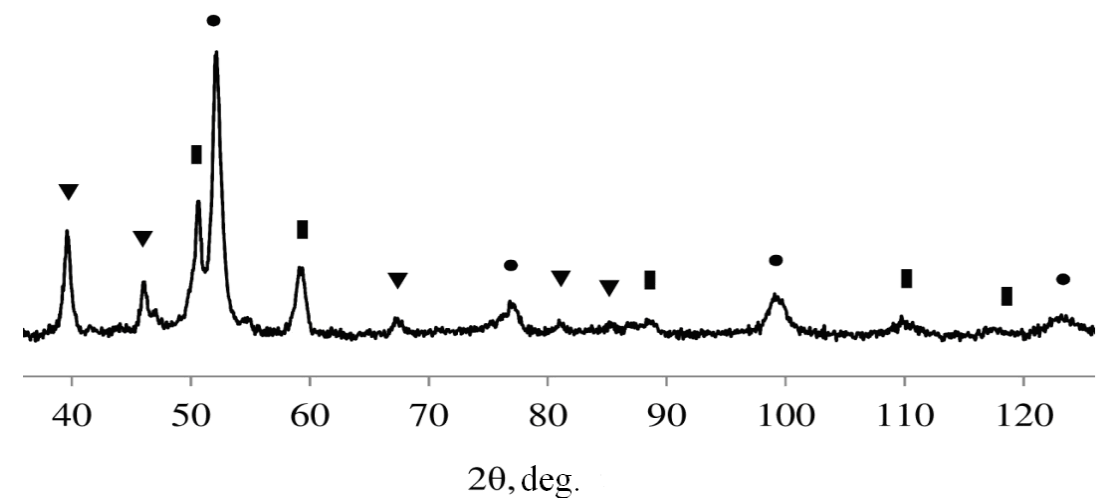

Fig. 3. Diffraction pattern $(\mathrm{CoK} \alpha)$ of the sample surface (nitrided steel P6M5) after the deposition of the Zr coat and LHEB irradiation.

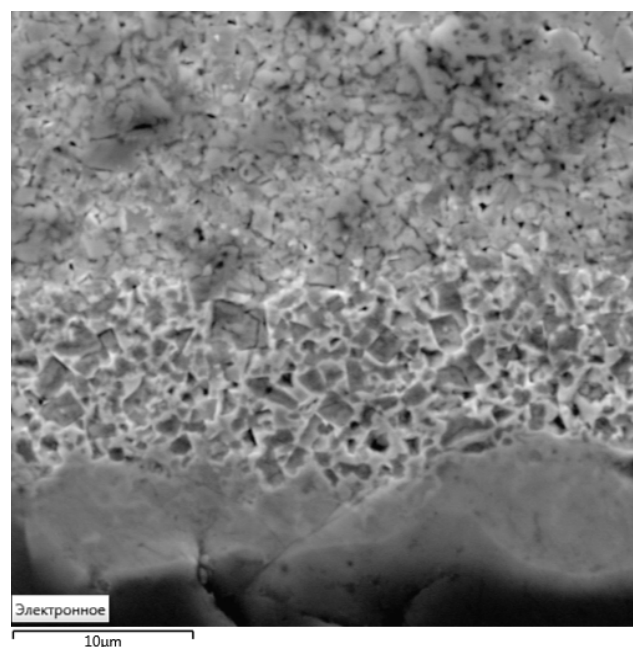

(a)

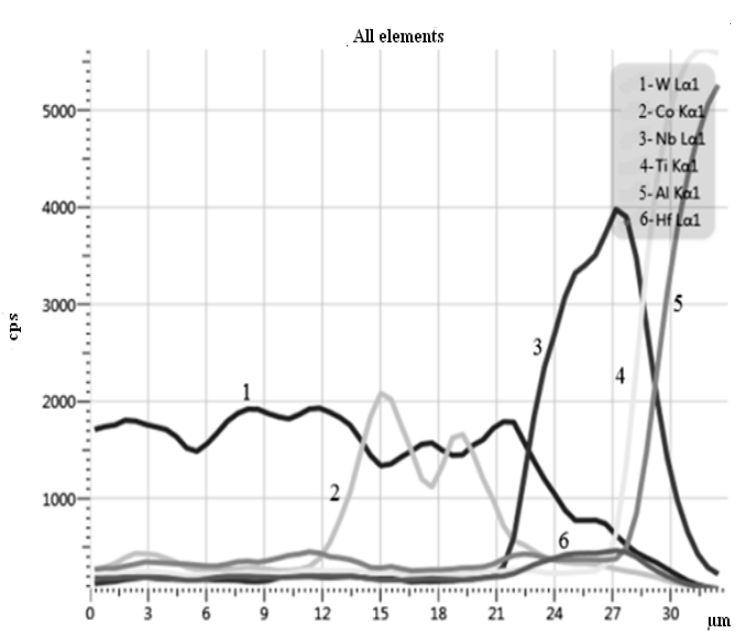

(b)

Fig. 4. (a) Image of an oblique surface of a plate of hardened alloy H13A (Sandvik Coromant) treated using LHEB with applied top coating $(\mathrm{TiAl}) \mathrm{N}$ in secondary electrons (electrolytic etching in a solution of oxalic acid), (b) distribution of chemical elements in the surface layer (top to bottom). 


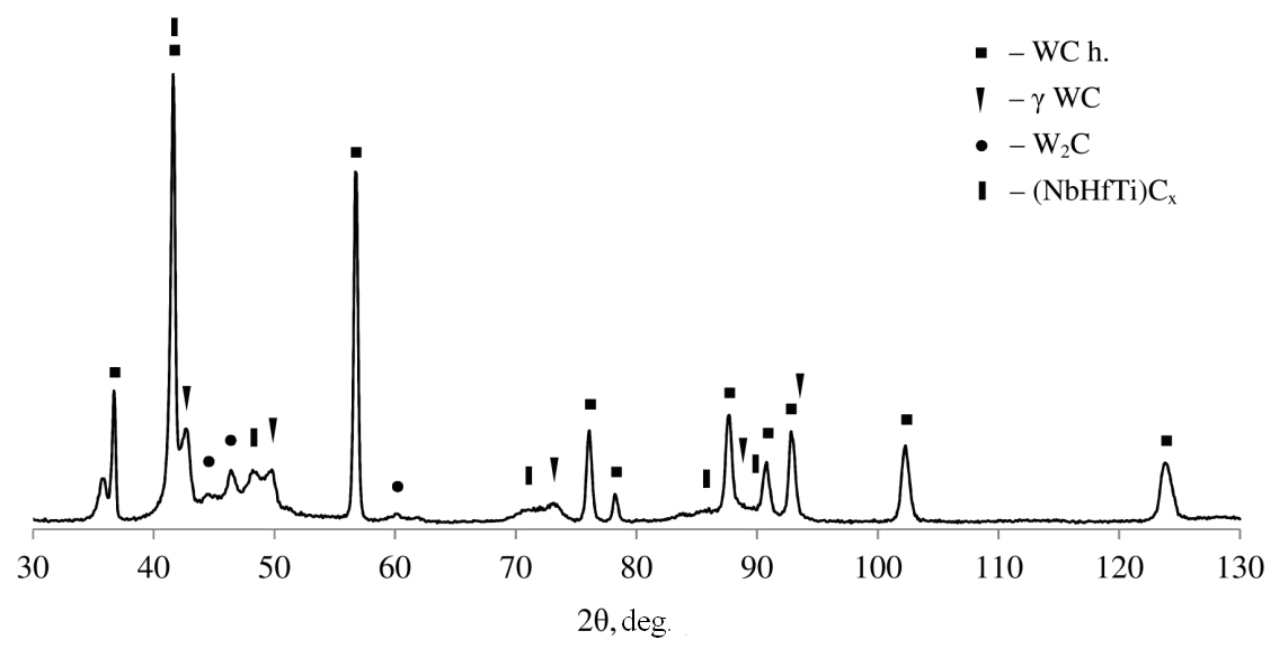

Fig. 5. Diffraction pattern $(\mathrm{CoK} \alpha)$ from the surface of H13A plate after surface modification with NbHfTi alloy.

Applying a thin layer of carbide-forming elements on the surface of the tool before processing by an electronic beam allows receiving multiphase carbide structure at the expense of microalloying at course of SHS reaction. Thus it is possible to avoid accumulation of free carbon on borders of carbide grains and to prevent cracking of a surface because of residual thermal tension. The external layer is enriched with MS-type refractory carbide phases, which, due to extremely high cooling rate, remain small and homogeneously distributed in the final product.

The powerful thermal impact of LHEB to the surface of the hard alloy coated in NbHfTi with thickness of about $200 \mathrm{~nm}$ with power density of the discharge about $5{\mathrm{~J} . \mathrm{cm}^{-2}}^{-2}$ and pulse duration of about $5 \mu$ s leads to the formation of face-centered cubic carbide phase, identified as $(\mathrm{Nb}, \mathrm{Hf}, \mathrm{Ti}) \mathrm{Cx}$. The reflections of $\gamma$-WC phase with lattice period $4.24 \times 10^{-1} \mathrm{~nm}$ are clearly visible.

Noteworthy is the same type of structure of niobiumbased carbide and high-temperature tungsten carbide. The corresponding diffraction pattern is presented in Figure 5.

\section{Conclusion}

The experimental results indicate the possibility of obtaining layers modified by microalloying on the surface of tool materials, which, due to the increased thermal stability of nonequilibrium structural and phase states, provide unique physical and mechanical properties.

Acknowledgements. The work was performed within the framework of the Resolution of the Government of the Russian
Federation dated 9 April 2010 No. 220 "On measures taken to attract key scientists to Russian educational institutions of higher professional education and scientific establishments of state academies of science and state research centers of the Russian Federation" (Agreement No. 14.B25.31.0012 dated 26 June 2013). The work is carried out on the equipment of the Center of collective use of MSTU "STANKIN" with financial support of the Ministry of Education and Science of Russian Federation, the Agreement No. 14.593.21.0004 of 04.12.2014, the unique identifier of the project RFMEFI59314X0004.

\section{References}

[1] S.V. Fedorov, M.D. Pavlov, A.A. Okunkova, Effect of structural and phase transformations in alloyed subsurface layer of hard-alloy tools on their wear resistance during cutting of high temperature alloys, J. Friction Wear 34 (2013) 190-198

[2] A.S. Rogachev, A.S. Mukasyan, Combustion for material synthesis. NY, CRC Press Reference, 2014, p. 424

[3] S.N. Grigoriev, A.S. Metel, S.V. Fedorov, Modification of the structure and properties of high-speed steel by a combined vacuum-plasma treatment, Metall. Heat Treatment Metals 1 (2012) 8-12

[4] A.B. Markov, A.V. Mikov, G. Ye. Ozur, A.G. Padej, Unit RITM-SP for the formation of surface alloys, Devices Experimental Techniques 6 (2011) 122-126

[5] A.B. Markov, E.V. Yakovlev, V.I. Petrov, Formation of Surface Alloys with a Low-Energy High-Current Electron Beam for Improving High-Voltage Hold-Off of Copper Electrodes, IEEE Trans. Plasma Sci. 41 (2013) 2177-2182 